\title{
Evolution des taux de croissance juvénile d'espèces forestières sous trois régimes expérimentaux de compétition
}

\author{
P. BELLEFLEUR et M. VILLENEUVE (*) \\ Département des sciences forestières \\ Faculté de foresterie et géodésie, Université Laval, Québec \\ Québec, Canada G1K 7P4
}

\begin{abstract}
Résumé
En étudiant l'évolution des taux de croissance en diamètre et en hauteur au cours des premières années de la succession secondaire, on a déterminé que le bouleau jaune (Betula alleghaniensis Britton) est l'espèce commerciale la plus compétitive et la mieux adaptée au site à ce stade de la succession. L'érable à sucre (Acer saccharum Marsh.) est globalement le moins efficace, le hêtre à grandes feuilles (Fagus grandifolia Ehrh.) se montrant un peu plus compétitif que lui. Cette croissance juvénile très rapide semble confirmer la dominance passagère du bouleau jaune dans la succession et laisse présager sa régression au profit de l'érable à sucre pour reformer la communauté originale d'avant la coupe, l'érablière à bouleau jaune et hêtre à grandes feuilles. Il est donc peu probable que les tendances observées actuellement se poursuivent jusqu'au stade final.
\end{abstract}

\section{Introduction}

\subsection{Problématique}

En foresterie, la coupe est le principal outil permettant de renouveler les peuplements (Sмiтh, 1962). Elle permet de créer les espaces nécessaires à l'établissement d'une nouvelle communauté. Les conditions existantes à ce moment déterminent non seulement les espèces qui s'y établiront mais aussi leur taux de croissance (Berglund, 1976). D’après Grime (1979), de petites différences au niveau de la croissance en hauteur exercent de profondes influences sur la compétitivité d'un semis pour l'utilisation de la lumière. Ce facteur est considéré comme le plus critique par BERGLUND (1976). Le même auteur, citant plusieurs études, rappelle que l'aptitude à la compétition et au maintien de la dominance sur un site particulier dépend

$\%$ Adresse actuelle du second auteur : Department of Forest Resources, University of New-Brunswick, Bag Service 44555, Fredericton, New-Brunswick, Canada E3B 6C2. 
avant tout du degré auquel les ressources du site satisfont les besoins de la plante et de l'efficacité de la plante à utiliser ces ressources par rapport à ses compétiteurs.

Les espèces qui formeront le peuplement final seront donc celles montrant la plus grande efficacité dans l'utilisation du site. Cette efficacité est quantifiable en terme de taux de croissance. La croissance en hauteur est très sensible aux variations de qualité du site, tandis que la croissance en diamètre est fortement reliće au degré de compétition (Davis, 1966). Bellefleur \& Larocoue (1983 a) et Bellefleur \& PÉTILLON (1983) concluent également que l'accroissement en diamètre est plus affecté par la compétition pour l'espace et le rayonnement solaire que l'accroissement en hauteur.

On peut en déduire sommairement que (1) le taux de croissance en hauteur est indicateur de la qualité du site pour l'espèce concernée; (2) le taux de croissance en diamètre traduit la compétitivité de l'espèce pour l'espace et le rayonnement solaire sur le site; (3) l'efficacité globale d'une espèce sur un site donné dépend à la fois de ses taux de croissance en hauteur et en diamètre, par rapport aux autres espèces.

\subsection{Buts et objectifs}

Cette étude veut déceler les différences existantes entre 3 espèces dites commerciales, l'érable à sucre (Acer saccharum Marsh.), le bouleau jaune (Betula alleghaniensis Britton) et le hêtre à grandes feuilles (Fagus grandifolia Ehrh.) au niveau de leurs taux de croissance en diamètre et en hauteur. Les objectifs sont (1) de comparer les taux annuels pour chaque espèce d'une année à l'autre; et (2) de vérifier l'in. fluence de l'élimination expérimentale de certains compétiteurs sur ces taux de croissance. On espère ainsi déterminer l'espèce la mieux adaptée au site et l'efficacité relative de chacune des 3 espèces ligneuses commerciales sur ce site, à ce stade de la succession secondaire.

\section{Matériel et méthode}

\subsection{Lieu d'étude}

La Station forestière de Duchesnay se situe à $71^{\circ} 42^{\prime} \mathrm{O}$ de longitude et $46^{\circ} 53^{\prime} \mathrm{N}$ de latitude, soit à $30 \mathrm{~km}$ à l'ouest de la ville de Québec (Canada), à la limite sud de la section forestière laurentienne L. 4 a (Rowe, 1972), dans le domaine de l'érablière à bouleau jaune (GrantNer, 1966). Le dispositif expérimental a été installé au printemps 1979 dans une bande coupée à blanc en janvier 1978 dans une ćrablière à bouleau jaune et hêtre à grandes feuilles (Bellefleur \& Pétul.oon, 1983). La superficie coupée mesure $100 \mathrm{~m}$ de largeur sur $400 \mathrm{~m}$ de longueur sur une pente de $16^{\circ}$ exposée au nord-est (Robitarlle, 1977).

Selon la nomenclature de la Commission canadienne de pédologie (ANONyme, 1978), le dépôt de surface est un till mince $(0,5 \mathrm{~m})$ à texture de sable loameux (75 p. 100 de sable, 21 p. 100 de limon et 4 p. 100 d'argile), à forte pierrosité et 
dont le drainage est bon. L'humus est de type mor et recouvre un podzol ferrohumique orthique (Bellefleur \& Pétillon, 1983). Les relevés faits par BLLlefteur \& PétIllon (1983) du 15 mai au 15 septembre 1980 indiquent une tcmpérature moyenne mensuelle de $15,4^{\circ} \mathrm{C}$, avec un maximum de $23,4^{\circ} \mathrm{C}$ et un minimim de $7,4^{\circ} \mathrm{C}$. Durant cette période, les précipitations ont totalisé $431,6 \mathrm{~mm}$.

\subsection{Dispositif expérimental}

Le dispositif mesure $18 \mathrm{~m}$ sur $90 \mathrm{~m}$ et est orienté perpendiculairement à la bande de coupe. Il comprend 180 parcelles de $3 \mathrm{~m}$ de côté, parmi lesquelles 90 ont été choisies au hasard pour faire l'objet des traitements. Les 30 premières demeurent à l'état naturel (témoin). Le deuxième tiers subit l'éradication des ligneux non commerciaux, i.e. autres que l'érable à sucre, le bouleau jaune, et lè hêtre à grandes feuilles, en coupant au niveau du collet les individus les plus grands et en arrachant les autres (traitement 1). Le dernier tiers voit éliminer les ligneux non commerciaux et les plantes herbacées (traitement 2). Les traitements sont effectués annuellement, au début et au milieu de la saison de végétation. Des places-échantillon de $1 \mathrm{~m}^{2}$ font l'objet de mesures et d'observations; elles se situent au centre des parcelles de $9 \mathrm{~m} 2$ auxquelles le traitement est appliqué, ceci afin d'éliminer les effets de bordure.

\subsection{Mesure des semis}

Dans toutes les places-échantillon, chaque semis, à l'exclusion des drageons et des rejets de souche, porte une étiquette pour permettre de suivre sa croissance réelle. Le diamètre en direction est-ouest est mesuré au collet à l'aide d'un micromètre, avec une précision de $0,1 \mathrm{~mm}$. La hauteur est mesuréc au millimètre près avec une règle, à partir d'un point de référence fixe au niveau du collet. Après la mesure initiale au printemps de 1979 , les semis ont été mesurés à chaque automne. Toutefois, des mesures printanières ont permis de relever les dommages causćs pendant l'hiver (gel, bris, mortalité). La densité totale des semis de toutes les espèces commerciales et non commerciales au printemps de 1979 était de $159 \times 10^{3}$ ha dont 39 p. 100 d'érable à sucre, 25 p. 100 de hêtre à grandes feuilles et 17 p. 100 de bouleau jaune; la variation annuelle a été présentée précédemment (BELLEFLEUR \& Pétillon, 1983).

\subsection{Hypothèses et traitement des données}

Les hypothèses de base à vérifier sont les suivantes : (1) pour une même cspèce, les taux de croissance en diamètre et en hauteur varient d'une année à l'autre; (2) pour une même année, les taux de croissance en diamètre ct en hauteur varient d'une espèce à l'autre; (3) pour une même année et une même espèce, les taux de croissance en diamètre et en hauteur varient d'un traitement à l'autre.

Les données originelles de taux de croissance ont nécessité une transformation logarithmique $\left[Y=\log _{10}(1+X)\right]$ pour normaliser leur distribution. L'homogénéité 
des variances a été vérifiée à l'aide du test de Bartlett avant de procéder à l'analyse de variance (SOKal \& Rohlf, 1981). La comparaison des moyennes a été faite au moyen du test de Duncan.

\section{Résultats et discussion}

\subsection{Espèces traitées}

En plus des 3 espèces dont les accroissements ont été suivis, 17 autres espèces vasculaires ont fait l'objet des traitements (tabl. 1). Les ligneux non commerciaux, éliminés dans les parcelles des traitements 1 et 2 , comprenaient 7 espèces. Les plantes herbacées, éliminées dans les parcelles du traitement 2 seulement, comptaient 10 espèces. De toutes ces espèces, 3 seulement sont pionnières : le cerisier de Pennsylvanie, le peuplier faux-tremble et le framboisier. Par ailleurs, toutes les espèces de la communauté climacique d'avant la coupe rase (l'érablière à boulcau jaune et hêtre à grandes feuilles) sont déjà en place; c'est donc dire que la coupe rase effectuée sur une aussi petite superficie $(100 \mathrm{~m} \times 400 \mathrm{~m})$ ne produit pas de retour en arrière brutal dans la succession forestière, mais laisse la surface coupée à l'état climatique avec l'ajout de quelques espèces pionnières qui disparaîtront rapidement.

\subsection{Variations temporelles des taux de croissance}

Le tableau 2 résume l'évolution des taux de croissance (en diamètre et en hauteur) d'année en année pour chaque espèce ; les taux sont exprimés en pourcentage de l'accroissement annuel sur le diamètre (ou la hauteur) total. L'analyse de variance décèle des différences significatives dans la plupart des cas. La première hypothèse est donc acceptée. On constate en $1^{\text {wr }}$ lieu que le hêtre à grandes feuilles et l'érable à sucre se distinguent du bouleau jaune. La tendance générale est, pour les 2 premiers, d'atteindre leurs maximums d'efficacité de croissance après un délai d'un an, en 1980, tandis que le bouleau jaune connaît sa croissance maximale dès l'année du traitement, en 1979. La prédétermination de la croissance durant l'année de formation des bourgeons explique ce phénomène. Kozlowskı (1964) insiste sur l'importance de cette prédétermination, citant plusieurs études démontrant une meilleure corrélation de la croissance en hauteur avec la saison de formation du bourgeon qu'avec la saison d'élongation de ce dernier, ce qui empêcherait l'érable à sucre et le hêtre à grandes feuilles de réagir dès l'année du traitement. Les résultats de MARks (1975) corroborent ceux du tableau 2 : l'érable à sucre et le hêtre à grandes feuilles sont des espèces à croissance prédéterminée tandis que le bouleau jaune possède une croissance indéterminée ; du point de vue de la prédétermination de la croissance, l'érable à sucre et le hêtre à grandes feuilles seraient plus probablement des espèces climaciques tandis que le bouleau jaune serait une espèce de transition dans la succession, ce qui est confirmé ici par sa croissance juvénile plus rapide. 


\section{TableaU 1}

Liste des espèces retrouvées sur le dispositif expérimental en coupe rase.

List of species found in the experimental design on the clear-cut area.

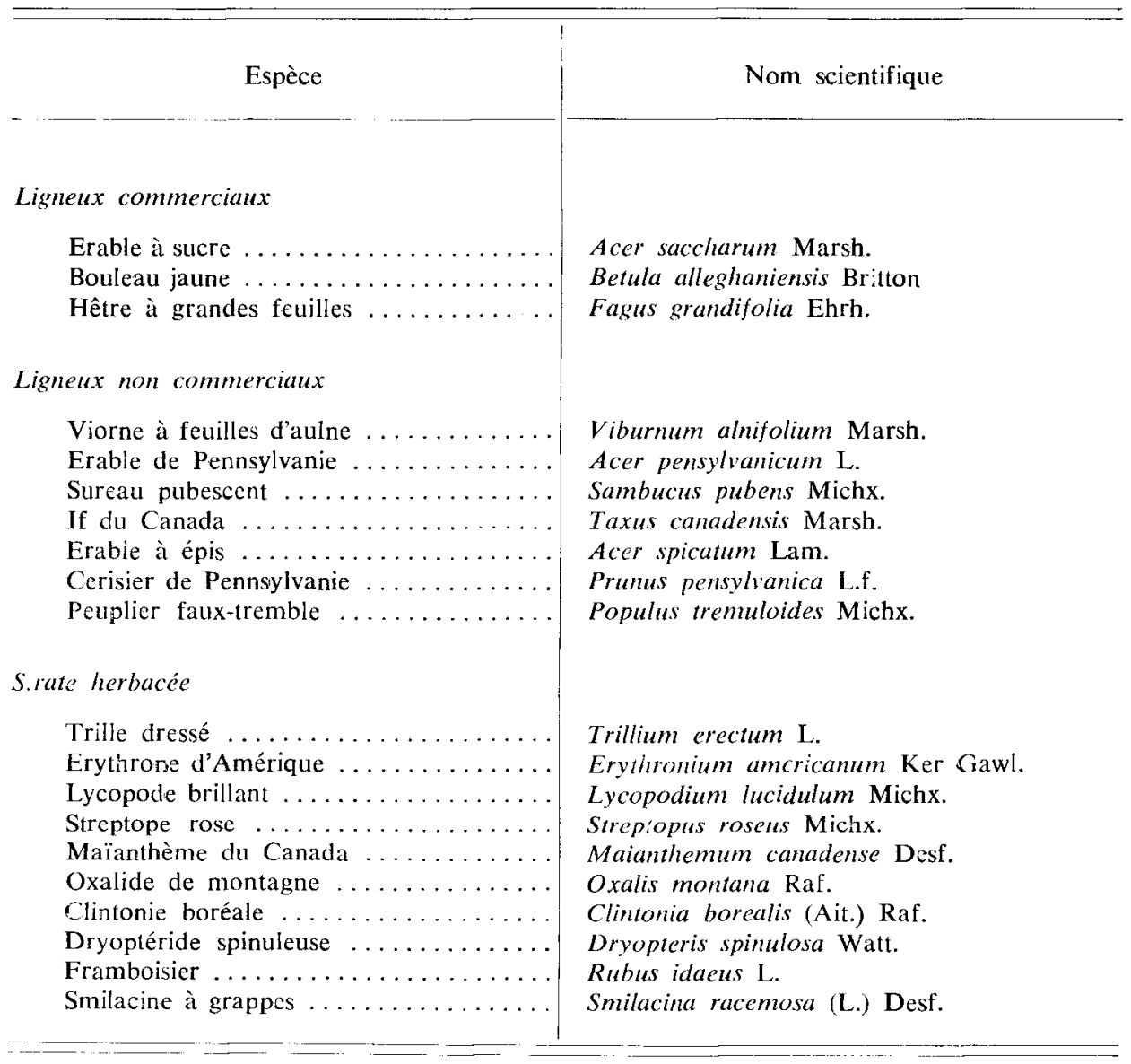

La réduction progressive d'année en année des taux de croissance chez les 3 espèces semble un phénomène tout à fait normal. Bellefleur \& LARocoue (1983 a) et Bellefleur \& Pétillon (1983) montrent que la croissance (en diamètre et en hauteur) est affectée par la compétition et que cette compétition s'intensifie d'année en année. La réduction progressive des taux de croissance peut se voir sous 2 points de vue différents : (1) biologique, la compétition accrue réduit la croissance annuelle ; (2) mathématique, un même accroissement représente une proportion de plus en plus faible de la taille totale du semis. 


\section{Tableau 2}

Taux de croissance annuelle en diamètre et en hauteur selon les espèces; moyennes sans distinction de traitement (p. 100).

Diameter and height annual growth rate per species; means of all treatments confounded (p. 100$)$.

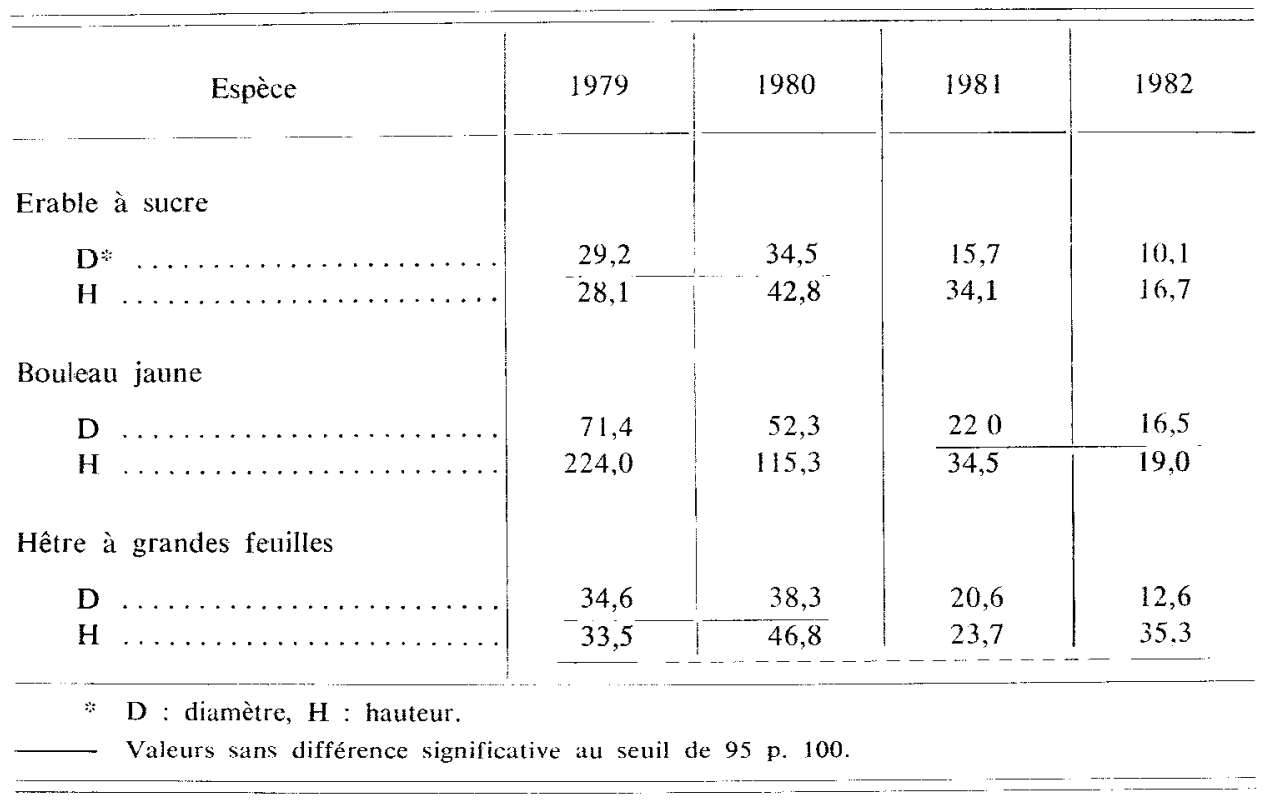

L'augmentation du taux de croissance en hauteur du hêtre à grandes feuilles pour 1982 pourrait être liée à une augmentation du recouvrement foliaire en 1981 . Bellefleur \& Larocoue (1983 b), Fowells (1965) et Logan (1973) s'accordent pour dire que les semis de hêtre à grandes feuilles ont une meilleure croissance sous couvert partiel. Les résultats de Bellefleur \& LARocove (1983 a, $1983 \mathrm{~b}$ ) font toutefois mention d'une adaptation tardive du hêtre à grandes feuilles suite aux traitements. La cause de cette augmentation de croissance demeure inconnue. Provient-elle de meilleures conditions de croissance en 1981 ou d'une mauvaise saison en 1980 ? Dans le $2^{\circ}$ cas, il faudrait plutôt parler de réduction du taux de croissance en 1981.

\subsection{Variations en fonction de l'espèce}

Le tableau 3 reprend les données du tableau 2, mais cette fois les analyses de variance portent sur les différences entre espèces pour une même année. A chaque année, au moins 1 espèce montre des différences significatives par rapport aux 2 autres, tant pour les accroissements en diamètre qu'en hauteur; la $2^{\text {e }}$ hypothèse est donc aussi acceptée. L'érable à sucre et le hêtre à grandes feuilles sont des 
espèces de climax, tolérantes à l'ombre et à croissance lente. Le boulcau jaune est classé comme espèce de transition, de tolérance modérée à l'ombre et à croissance plus rapide (Boivin, 1971; Fowells, 1965; Godman, 1957; Linteau, 1948 ; Logan, 1965, 1973; Marks, 1975; Spurr \& Barnes, 1980; Tubiss, 1973). Ceci concorde avec les résultats figurant au tableau 3. Toutefois, les travaux de BELLEFLEUR \& LAROCQUE (1983 b) démontrent que le bouleau jaune tolère mieux les conditions d'ombrage que l'érable à sucre et le hêtre à grandes feuilles durant les premières années suivant la germination. On constate de prime abord que le bouleau jaune s'avère le plus efficace sur ce site : ses taux de croissance cn diamètre et en hauteur sont toujours supérieurs ou égaux à ceux des 2 autres espèces (à l'exception de la hauteur en 1982). Le bouleau jaune est donc, à ce stade de la succession, le plus compétitif et le mieux adapté au site.

\section{TABleau 3}

Taux de croissance annuelle en diamètre et en hauteur selon les années; moyennes sans distinction de traitement (p. 100).

Diameter and height annual growth rate per year; means of all treatments confounded (p. 100$)$.

\begin{tabular}{|c|c|c|c|c|}
\hline & Année & Erable à sucre & Bouleau jaune & $\begin{array}{c}\text { Hêtre } \\
\text { à grandes feuilles }\end{array}$ \\
\hline \multirow[t]{4}{*}{ Diamètre } & 1979 & 29,2 & 71,4 & 34,6 \\
\hline & 1980 & 34,5 & 52,3 & 38,3 \\
\hline & 1981 & 15,7 & 22,0 & 20,6 \\
\hline & 1982 & 10,1 & 16,5 & 12,6 \\
\hline \multirow[t]{4}{*}{ Hauteur } & 1979 & 28,1 & 224,0 & 33,5 \\
\hline & 1980 & 42.8 & 115,3 & 46,8 \\
\hline & 1981 & 34,1 & 34,5 & 23,7 \\
\hline & 1982 & 16.7 & 19,0 & 35,3 \\
\hline
\end{tabular}

L'érable à sucre el le hêtre à grandes feuilles montrent des taux de croissance en diamètre assez comparables durant les 2 premières années. Par la suite, le hêtre à grandes feuilles semble micux supporter la compétition (taux de croissance en diamètre supérieur à celui de l'érable à sucre). Au niveau des taux de croissance en hautcur, à l'exception de 1981, lc hêtre à grandes feuilles se montre mieux adapté au site que l'érable à sucre, espèce très exigeante. 


\section{TABLEAU 4}

Taux de croissance annuelle en diamètre et en hauteur selon les espèces;

moyennes pour chaque traitement (p. 100).

Diameter and height annual growth rate per species; means for each treatment.

\begin{tabular}{|c|c|c|c|c|c|c|c|}
\hline \multirow{2}{*}{ Espèce } & \multirow{2}{*}{ Année } & \multicolumn{3}{|c|}{ Damètre } & \multicolumn{3}{|c|}{ Hauteur } \\
\hline & & $t^{*}$ & $\mathrm{~T}_{1}$ & $\mathrm{~T}_{2}$ & $t$ & $\mathrm{~T}_{1}$ & $\mathrm{~T}_{2}$ \\
\hline \multirow{4}{*}{$\begin{array}{l}\text { Erable } \\
\text { à sucre }\end{array}$} & 1979 & 31,6 & 37,3 & 24,5 & 25,5 & 40,2 & 25,5 \\
\hline & 1980 & 26,2 & 35,4 & 45,5 & 35,8 & 43,2 & 56,5 \\
\hline & 1981 & 12,8 & 17,4 & 21,6 & 31,3 & 31,4 & 44,0 \\
\hline & 1982 & 11,5 & 9,5 & 12,4 & 19,9 & 17,6 & 16,0 \\
\hline \multirow[t]{4}{*}{ Bouleau jaune } & 1979 & 120,8 & 68,7 & 50,2 & 546,4 & 221,1 & 101,8 \\
\hline & 1980 & 47,3 & 46,7 & 83,7 & 132,7 & 108,4 & 182,8 \\
\hline & 1981 & 18,2 & 19,9 & 34,8 & 24,4 & 28,8 & 65,7 \\
\hline & 1982 & 15,7 & 18,5 & 17,7 & 16,7 & 15,3 & 28,7 \\
\hline \multirow{4}{*}{$\begin{array}{c}\text { Hêtre } \\
\text { à grandes } \\
\text { feuilles }\end{array}$} & 1979 & 33,1 & 43,1 & 29,5 & 35,0 & 37,2 & 30,7 \\
\hline & 1980 & 30,1 & 42,3 & 49,5 & 43,1 & 52,7 & 48,7 \\
\hline & 1981 & 15,8 & 24,9 & 25,7 & 18,1 & 25,7 & 39,5 \\
\hline & 1982 & 12,2 & 14,1 & 14,8 & 32,1 & 36,8 & 42,1 \\
\hline
\end{tabular}

$\because t$ : témoin, $T_{1}:$ traitement $1, T_{2}:$ traitement 2.

Valeurs sans différence significative au seuil de 95 p. 100. 


\subsection{Effets des traitements}

Unc dernière série d'analyses confirme la $3^{\circ}$ hypothèse (tabl. 4). Pour chaque espèce et chacune des 3 premières années, l'un des traitements est significativement différent des 2 autres, tant pour les accroissements en diamètre qu'en hauteur. Les traitements expérimentaux ont donc une influence significative sur les taux de croissance. La transformation logarithmique a pu homogénéiser les variances pour la moitié des analyses. De nouveau, on constate une réduction progressive des taux de croissance depuis 1979 pour l'ensemble des traitements. En général, ces taux sont plus élevés pour les parcelles du traitement 2 et plus faibles pour les parcelles témoins. Ceci est en accord avec les résultats de Bellefleur \& LARocoue (1983 a) et de Bellefleur \& PÉtillon (1983) concernant les accroissements annuels. La compétition de la part des ligneux non commerciaux et des herbacées est donc statistiquement et biologiquement très importante. L'effet des traitements sur la compétitivité des ligneux commerciatx et sur leur efficacité dans l'utilisation du site devient négligeable en 1982 : l'analyse statistique ne révèle aucune différence significative entre les traitements pour cette année.

Les parcelles du traitement 2 ayant été traitées tardivement en 1979 (juin), les taux de croissance doivent être comparés à ceux des parcelles du traitement 1, dans le cas du bouleau jaune. L'excellente performance de celui-ci dans les parcelles témoins en 1979 ne s'explique, statistiquement, que par l'effet de hasard, c'est-à-dire par des causes autres que les traitements. Bellefleur \& Pétrllon (1983) signalent que le bouleau jaune se retrouve surtout en petites colonies et que la distribution des semis (toutes espèces) est irrégulière suivant les traitements. Pour l'érable à sucre et le hêtre à grandes feuilles, à croissance prédéterminée, ce traitement tardif n'a pas empêché une meilleure performance en 1980 par rapport aux autres parcelles. Le bourgeon terminal n'est formé que vers la fin de la saison de croissance, saison qui se poursuit jusqu'en septembre (Gregory, 1980; Kozlowski \& Ward, 1957).

\section{Conclusion}

Cette étude a permis de déceler des différences significatives de taux de croissance en diamètre et en hauteur, entre l'érable à sucre, le bouleau jaune et le hêtre à grandes feuilles. On a pu déterminer quelle espèce est la mieux adaptée au site et quelle est l'efficacité relative de ces 3 espèces à ce stade de la succession secondaire.

Nous avons déterminé que le bouleau jaune avait une réponse immédiate aux traitements dès 1979, contrairement à l'érable à sucre et au hêtre à grandes feuilles. De plus, le bouleau jaune présente les meilleurs taux de croissance (tant en diamètre qu'en hauteur), tandis que l'érable à sucre est le moins performant. Le hêtre à grandes feuilles montre un rendement semblable à celui de l'érable à sucre mais semble mieux supporter la compétition. Finalement, l'influence significative des traitements sur les taux de croissance indique que chacune des 3 espèces est affectée par la compétition de la part des ligneux non commerciaux et des herbacées. Cette influence des traitements sur les taux de croissance semble s'estomper at fil des ans. 
Si les tendances actuelles se poursuivaient, il semble que la communauté serait dominée par le bouleau jaune, et que l'érable à sucre et le hêtre à grandes feuilles seraient sous-dominants dans des proportions à peu près égales. Toutefois, les relevés de GrandTner (1966) dans l'érablière à bouleau jaune et hêtre à grandes feuilles indiquent que c'est l'érable à sucre qui est normalement le plus abondant, avec un peu plus de bouleau jaune que de hêtre à grandes feuilles. Selon Spurr \& Barnes (1980), l'érable à sucre peut devenir l'espèce dominante grâce à sa très grande tolérance à l'ombre et à une production imposante de graines qui se dispersent et germent aisément. Godman (1957), HetT \& Loucks (1971) ainsi que Fowells (1965) le considèrent aussi comme l'un des arbres les plus tolérants à l'ombre.

En regard de ces informations, la performance exceptionnelle de la croissance juvénile du bouleau jaune confirme son rôle d'espèce de transition et il est douteux que sa dominance observée en ce début de succession se poursuive jusqu'au stade final. Vraisemblablement, l'érable à sucre reprendra la dominance sur ce site. Il sera donc très intéressant d'observer ce qui adviendra du bouleau jaune dans les années à venir. Sans doute que par sa faible dispersion sur le terrain et son habitude à former de petites colonies, il s'éliminera de lui-même par compétition intraspécifique puisque le plus proche compétiteur d'un bouleau jaune est très souvent, forcément, un autre individu de la même espèce. Par la suite, l'érable à sucre qui est beaucoup plus longévive assurera la dominance et la conservera grâce à son aptitude à se reproduire à l'ombre. Le bouleau jaune se maintiendra dans la communauté en plus faible densité en se reproduisant dans les ouvertures naturelles.

\section{Remerciements}

Nous tenons à remercier chaleureusement le $\mathrm{D}^{r}$ Miroslav M. Grandtner et M. Rosaire Jean, tous deux du Laboratoire d'écologie forestière de l'université Laval (Québec, Canada), le $\mathrm{D}^{r}$ Jorge P. Cancela da Fonseca, du Laboratoire de biologie végétale et d'écologie forestière de l'université Paris VII (Fontainebleau, France) et M. Guy Larocque de l'Institut forestier national de Petawawa (Canada) qui ont relu et critiqué notre manuscrit. Nous sommes reconnaissants envers $\mathbf{M}^{\text {11u }}$ Hélène Boisvert qui a soumis notre manuscrit sous système de traitement de texte. Enfin, nous remercions sincèrement le Conseil de recherche en sciences naturelles et en génie du Canada pour le financement de cette étude et pour l'octroi d'une bourse d'été au second auteur.

\section{Summary}

Modification of early successionnal growth rates of forest species under three experimental competition regimes.

Measurements of diameter and height growth at early stages of secondary succession have shown that yellow birch (Betula alleghamiensis Britton) is the most suited commercial species on its site at this stage of succession. Sugar maple (Acer saccharun Marsh.) is globally the less efficient, while american beech (Fagus grandifolia Ehrh.) is slightly more competitive. Its very high early growth seems to confirm the temporary dominance of ycllow birch in succession and leads to believe in its decrease to the benefit of sugar maple to rebuild the original sugar maple stand with yellow birch and american beech. It is therefore unlikely that the actual trend will persist until climax. 


\section{Références bibliographiques}

AnONYme, 1978. Le système de classification des sols. Ministère de l'agriculture du Canada, Publ. 1646.

Bellefleur P., Larocque G., 1983 a. Compétition pour le rayonnement solaire en début de succession secondaire dans une érablière à bouleau jaune et hêtre. Can. J. For. Res., 13 (3), 514-521.

Bellefleur P., Larocque G., 1983 b. Comparaison de la croissance d'espèces ligneuses en milieu ouvert et sous couvert forestier. Can. J. For. Res., 13 (3), 508-513.

Belleflevr P., PÉtillon Y., 1983. Expérimentation sur la compétition interspécifique par ćlimination sélective d'espèces forestières. Can. J. For. Res., 13 (3), 522-532.

Berglund J.V., 1976. Silvics. State University of New York, College of Environmental Science and Forestry, Syracuse, 448 p.

BoIvin J.L., 1971. A study of regeneration after clear felling hardwood and mixed stands in Quebec. For. Chron., 47 (2), 82-85.

Davis K.P., 1966. Forest management : regulation and valuation. McGraw-Hill Book Company, 2nd ed., New York, 519 p.

Fowells H.A., 1965. Silvics of forest trees of the United States. U.S.D.A., Washington, D.C. Agric. Hdbk no. 271,762 p.

Godman R.M., 1957. Silvical characteristics of sugar maple. U.S. Forest Serv., Lakc Statcs For. Exp. Sta., no. 50, 24 p.

Grandiner M.M., 1966. La végétation forestière du Québec méridional. Presses de l'Univ. Laval, Québec, 216 p.

Gregory R.A., 1980. Annual cycle of shoot development in sugar maple. Can. J. For. Res., 10 (3), 316-326.

Grim: J.P., 1979. Plant strategies and vegetation processes. J. Wiley and Sons, Ltd., Toronto, $222 \mathrm{p}$.

Hext J.M., Loucks O.L., 1971. Sugar maple (Acer saccharum Marsh.) seedling mortality. J. Ecol., 59 (2), 507-520.

KozLOWSKr T.T., 1964. Shoot growth in woody plants. Bot. Rev., 30 (3), 335-392.

KOZLOWSKI T.T. and R.C. WARD, 1957. Seasonal height growth of deciduous trees. For. Sci., 3, 168-174.

LiNTEAU A., 1948. Factors affecting germination and yearly survival of ycllow birch in Quebec. For. Chron., 24, 27-86.

LOGAN K.T., 1965. Growth of tree seedlings as affected by light intensity. I. - White birch, yellow birch, sugar maple, and silver maple. Can. Dept. Forest., no. 1121 , $16 \mathrm{p}$.

LOGAN K.T., 1973. Growth of tree seedlings as affected by light intensity. V. White ash, beech, eastern hemlock and general conclusions. Dept. Environ., Can. For. Serv., no. 1323,12 p.

MARKS P.L., 1975. On the relation between extension growth and successionnal status of deciduous trees of the NE U.-S. Bull. Torrey Bot. Club, 102 (4), 172-177.

Robitaille L., 1977. Recherches sylvicoles sur les feuillus nordiques à la Station forestière de Duchesnay. For. Chron., 53 (4), 201-203.

Rowe J.S., 1972. Les régions forestières du Canada. Ministère de l'Environnement, Service canadien des forêts. Publ., n" 1300 (en français).

Sмiтh D.M., 1962. The practice of sylviculture. J. Wiley and Sons, N.Y., 7th ed., 578 p.

SOKAL R.R., RoHLF F.J., 1981. Biometry. The principles and practice of statistics in biological research. W.H. Freeman and Co., San Francisco, 2nd ed., 859 p.

Spurr S.H., Barnes B.V., 1980. Forest ecology. J. Wiley and Sons, N.Y., 3rd ed., 687 p.

TubBs C.H., 1973. Allelopathic relationship between yellow birch and sugar maple seedlings. For. Sci., 19 (2), 139-145. 